\title{
Position Sense Deficits at the Lower Limbs in Early Multiple Sclerosis: Clinical and Neural Correlates
}

Neurorehabilitation and

Neural Repair

$\mid-11$

C) The Author(s) 2020

Article reuse guidelines:

sagepub.com/journals-permissions

DOI: I0.1 I77/I545968320902/26

journals.sagepub.com/home/nnr

@SAGE

\author{
Riccardo landolo, PhD 1,2,*, Giulia Bommarito, MD ${ }^{3, *}$ (D), Laura Falcitano, MD 3 , \\ Simona Schiavi, PhD $^{3}$ (D), Niccolò Piaggio, MD $^{3}$, Giovanni Luigi Mancardi, MD ${ }^{3}$, \\ Maura Casadio, $\mathbf{P h D}^{1,2, \dagger}$, and Matilde Inglese, $\mathbf{P h D}^{3,4, \dagger}$
}

\begin{abstract}
Background/Objective. Position sense, defined as the ability to identify joint and limb position in space, is crucial for balance and gait but has received limited attention in patients with multiple sclerosis (MS). We investigated lower limb position sense deficits, their neural correlates, and their effects on standing balance in patients with early MS. Methods. A total of 24 patients with early relapsing-remitting MS and 24 healthy controls performed ipsilateral and contralateral matching tasks with the right foot during functional magnetic resonance imaging. Corpus callosum (CC) integrity was estimated with diffusion tensor imaging. Patients also underwent an assessment of balance during quiet standing. We investigated differences between the 2 groups and the relations among proprioceptive errors, balance performance, and functional/ structural correlates. Results. During the contralateral matching task, patients demonstrated a higher matching error than controls, which correlated with the microstructural damage of the CC and with balance ability. In contrast, during the ipsilateral task, the 2 groups showed a similar matching performance, but patients displayed a functional reorganization involving the parietal areas. Neural activity in the frontoparietal regions correlated with the performance during both proprioceptive matching tasks and quiet standing. Conclusion. Patients with early MS had subtle, clinically undetectable, position sense deficits at the lower limbs that, nevertheless, affected standing balance. Functional changes allowed correct proprioception processing during the ipsilateral matching task but not during the more demanding bilateral task, possibly because of damage to the CC. These findings provide new insights into the mechanisms underlying disability in MS and could influence the design of neurorehabilitation protocols.
\end{abstract}

\section{Keywords}

proprioception, early multiple sclerosis, lower limbs, fMRI, balance, functional plasticity

\section{Introduction}

The ability to identify the position of lower limbs in space is critical for ambulation ${ }^{1}$ and balance control. ${ }^{1,2}$ As proprioception results from a complex process of integration of sensory inputs from different peripheral receptors, involving the central nervous system (CNS) at different levels, ${ }^{3}$ it is likely to be affected in a spatially disseminated disease such as multiple sclerosis (MS). MS is a chronic demyelinating disease of the CNS, causing neurological disability, especially in young adults. ${ }^{4}$ Sensory deficits are predominant in patients with MS, and among them, proprioceptive impairment is the most common, involving the lower more than the upper limbs. ${ }^{5,6}$ Moreover, patients with MS frequently have an impaired control of ankle motion, ${ }^{7}$ which is necessary for maintaining standing posture and during gait. ${ }^{8}$ Taken altogether, these observations suggest that sensorimotor impairment at the level of the ankle may be a primary factor that determines mobility and balance impairment in people with
'RBCS Department, Istituto Italiano di Tecnologia, Genova, Italy ${ }^{2}$ Department of Informatics, Bioengeneering, Robotics and System Engineering (DIBRIS), University of Genoa, Italy

${ }^{3}$ Department of Neuroscience, Rehabilitation, Ophthalmology, Genetics, Maternal and Child Health, (DINOGMI) University of Genova, Italy ${ }^{4}$ Ospedale Policlinico San Martino-IRCCS, Genova, Italy

*Riccardo landolo and Giulia Bommarito should be considered joint first authors.

†Maura Casadio and Matilde Inglese should be considered joint senior authors.

Supplementary material for this article is available on the Neurorehabilitation \& Neural Repair website at http://nnr.sagepub.com/ content/by/supplemental-data.

\section{Corresponding Author:}

Matilde Inglese, PhD, Department of Neuroscience, Rehabilitation, Ophthalmology, Genetics, Maternal and Child Health (DINOGMI), University of Genova, Largo Paolo Daneo 3, Genova, 16I32, Italy. Email: m.inglese@unige.it 
MS. However, the role of proprioception impairment in determining disability is often underestimated because of the limited sensitivity and reproducibility of the conventional clinical measures. ${ }^{9}$ Furthermore, in MS patients, the investigation of proprioception at the level of the lower limbs and specifically at the level of the ankle joints has received little attention so far, especially in terms of neural correlates. Indeed, previous studies focused on brain functional activity during active or passive motor tasks, including both upper limbs and ankle movements. ${ }^{10-12}$

It has been also reported that position sense at the level of the ankle, in terms of either behavioral outcome or neural activity, correlates with balance performance in both healthy subjects (HS) $)^{13,14}$ and patients with neurological diseases. ${ }^{15}$ Although balance impairment characterizes MS from its early stages ${ }^{16}$ and it has been extensively investigated, ${ }^{17-19}$ none of these studies explored the specific relationship between proprioceptive-related brain activity and balance ability during quiet standing in MS patients.

Finally, both the performance of sensory tasks requiring an interhemispheric transfer of information and postural control rely on the integrity of the corpus callosum (CC). ${ }^{20,21}$ The anterior part and midbody of the CC connect higher-order sensory and primary/secondary somatosensory areas, respectively, and $\mathrm{CC}$ integrity is required during bimanual coordination movements. ${ }^{22}$ The $\mathrm{CC}$ is affected from the early stages of $\mathrm{MS},{ }^{23}$ and its microstructural disruption has been associated with poor postural control. ${ }^{21}$ However, no study has examined the relationship between microstructural integrity of the $\mathrm{CC}$ and proprioceptive impairment during bilateral sensory tasks with the lower limbs or during quiet standing in patients at the early stages of the disease.

Against this background, we planned this study with 3 specific aims. The first aim was to assess distal lower limbs proprioception, in terms of both behavioral performance and neural activity, in patients with early relapsingremitting MS (eRR-MS). Specifically, we investigated the proprioceptive-related brain activity using functional MRI (fMRI) during ipsilateral and contralateral matching tasks ${ }^{24}$ performed with the lower limbs. The second aim of our study was to determine whether, in eRR-MS patients, the functional neural correlates of proprioception and behavioral performance during the matching tasks were related to postural control during quiet standing, thus investigating the impact of proprioceptive deficits on balance. Finally, as a third aim, we tested the hypothesis that the structural damage in the $\mathrm{CC}$ could affect the performance during both proprioceptive matching task requiring bilateral coordination and quiet standing, since the early stages of the disease.

\section{Material and Methods}

\section{Participants}

Outpatients with eRR-MS and gender- and age-matched HS were prospectively enrolled. The inclusion criteria were the following: (1) right or mixed foot dominance (Waterloo inventory score $>-6),{ }^{25}(2)$ no previous history of musculoskeletal injuries at the lower limbs level, (3) no previous intense performance of sport activities that involved the lower limbs extensively, and (4) no other neurological or psychiatric disorder. eRR-MS patients were selected according to the McDonald criteria $2017^{26}$ and with the following additional inclusion criteria: (5) disease duration of less than 5 years and (6) no relapses in the 3 months previous to the examination.

We screened 34 eRR-MS patients. However, 5 patients were excluded because they were left footed and 3 of them because they suffered a relapse before the examination. Of the 26 patients, 2 were excluded from the analysis: the first one because she did not complete the MRI scan, the second one because of the poor image quality resulting from motion artifacts. Thus, the final sample size of our study included 48 individuals: 24 with eRR-MS and 24 age- and gendermatched HS.

Patients with eRR-MS underwent clinical evaluation, including Expanded Disability Status Scale (EDSS) and assessment of the upright standing ability by means of a WII balance board, within 2 days of MRI acquisition.

The study conforms to the standard of the declaration of Helsinki and was approved by the local ethical committee (CER Liguria, No. 222REG2017). All participants provided written informed consent prior to participation in the study.

\section{fMRI Experimental Setup and Protocol}

For all participants, we evaluated the distal lower limb proprioception inside the magnetic resonance (MR) environment by using the MR-compatible setup described in Iandolo et $\mathrm{al}^{27}$ (see supplementary information [SI] and Figures 1A and 1B for further details). We asked the participants to perform ipsilateral and contralateral matching tasks with the right foot during fMRI. In addition, participants performed ipsilateral and bilateral active motor tasks involving the same dorsiplantar flexion movements needed to perform the matching tasks. In both the active motor tasks and the matching tasks, we used a block fMRI design with 4 blocks of rest ( $30 \mathrm{~s}$ each) alternated with 4 blocks of foot movements for the motor task and 6 blocks of rest alternated with 6 blocks of position matching for the proprioceptive task. All participants performed the following tasks (Figure 1D and SI for further details):

- Unilateral and bilateral active motor tasks (ACTIVEUNILAT and ACTIVE-BILAT, respectively). Partici- 


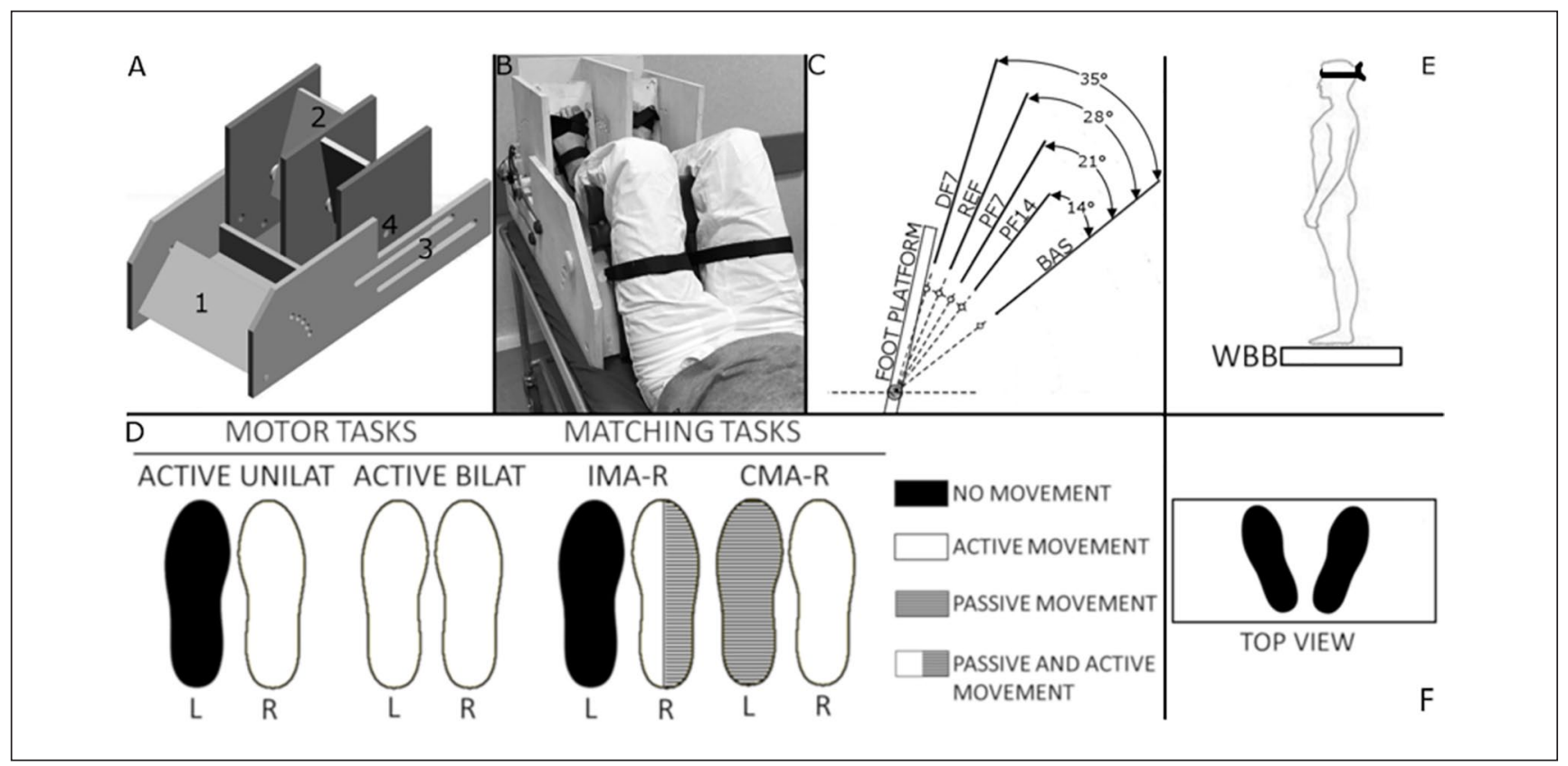

Figure I. A. Custom-made MR compatible passive device used during the magnetic resonance imaging (MRI) experimental protocol. The numbers indicate ( $I$ ) the adjustable tight platform; (2) the foot platforms that allow for each foot independently I degree-offreedom movement in the sagittal plane; (3) the 2 rails ( 2 per side) that allow the thigh platform and the foot platforms to slide relative to one another to fit different participants' anthropometries; (4) the MR compatible optical encoder was attached to each platform's axis of rotation. B. An overview of a participant outside the MR environment. The thighs and the feet were firmly strapped to the corresponding platform with VELCRO straps. C. The 4 presented platform positions (PFI4, PF7, REF, and DF7) that the participants were required to match during each matching task. The angle values on the right show the rotation values of the platform with respect to the BAS position from which each trial started. D. Task functional MRI protocols during motor and matching tasks. Rest and block duration was $30 \mathrm{~s}$ each. Tasks were randomized across patients. Overview of the patient and the feet position during the balance control task ( $E$ and F). Figure adopted with permissions from landolo et al. (landolo, Bellini and Saiote, 2018).

pants actively moved the right foot in the sagittal plane, during ACTIVE-UNILAT, or both feet in phase, during ACTIVE-BILAT, by synchronizing with a metronome set at $1 \mathrm{~Hz}$.

- Ipsilateral matching task with the right-dominant foot (IMA-R). The operator passively moved the foot from the starting position (Figure 1C, BAS) to one of the 4 target positions (Figure 1C) and then back to the BAS position. Subsequently, participants were asked to reach the target position with the same foot and then return to the BAS position.

- Contralateral matching task with the right-dominant foot (CMA-R). An operator moved the left nondominant foot to 1 of the same 4 positions as in the IMA-R task. The participant had to reach the selected position with the contralateral right-dominant foot and go back to the BAS location with both feet.

During the matching tasks, participants were required to keep their eyes closed. See SI for further details about matching task description and foot position during the tasks.

\section{Behavioral Analysis of the Matching Performance}

As an indicator of the matching performance, we computed the constant error (CE) - that is, the difference between the position reached by the matching foot and the target position, both measured in terms of angular rotations of the foot platforms. The values computed for each trial were averaged across repetitions and across targets, obtaining a single measure of the overall performance to correlate with the fMRI data in each task (IMA-R and CMA-R), with the structural damage of the $\mathrm{CC}$ as well as with the parameter assessing the balance performance. This measure indicates a systematic error: a negative value corresponds to an undershoot of the target positions, and a positive value to an overshoot; if the CE is zero, there is no systematic error. 


\section{Assessment of the Upright Standing Ability of eRR-MS Patients}

During the neurological examination, all eRR-MS patients underwent also a test aiming at evaluating their standing balance. They stand upright and barefoot on a Nintendo WII Balance Board (WBB, Nintendo, Kyoto, Japan), with the arms relaxed along the sides of the body, the heels separated by about $2 \mathrm{~cm}$, and the feet abducted at $20^{\circ}$ (see Figures $1 \mathrm{E}$ and $1 \mathrm{~F}) .^{28}$ They were asked to remain as still as possible for 3 trials, each lasting $40 \mathrm{~s}$. We asked participants to keep their eyes closed during the 3 testing trials to increase reliance on proprioception and to eliminate visual cues. ${ }^{13,18}$

Then, for each trial, we estimated the root mean square of the anteroposterior oscillations of the center of pressure $\left(\mathrm{RMS}_{\mathrm{AP}}[\mathrm{mm}]\right)$. We chose this parameter because it is influenced by the responses of the muscle spindle, and thus, it is strictly related to proprioceptive information; an increment of this parameter reflects poor balance performance. ${ }^{13}$ Moreover, $\mathrm{RMS}_{\mathrm{AP}}$ has been indicated to be among the most reliable parameters when using a WBB in HS and patients with MS. ${ }^{29}$ No learning effects were observed across the 3 trials and, as already described in a previous study ${ }^{28}$, we averaged the obtained values. See SI for additional information about data analysis from the WBB.

\section{MRI Acquisition and Image Processing}

All participants underwent MRI at $1.5 \mathrm{~T}$ Signa Excite (Signa Excite General Electric Healthcare, WI, USA) with 8 channels phased-array head coil. The protocol included the following: (1) high-resolution fast spoiled gradient echo 3D T1-weighted sequence, (2) spin-echo dual-echo proton density-weighted and T2-weighted sequence, (3) singleshot echo-planar imaging sequence for fMRI during active motor and matching tasks, and (4) an axial single-shot spin echo diffusion tensor imaging (DTI) sequence. MRI sequences details and fMRI and DTI data processing are described in SI.

\section{Statistical Analysis}

The statistical analysis was performed with SPSS (version 20.0), except for the fMRI section, performed with FSL. Prior to all statistical testing, we tested (Kolmogorov-Smirnov test) the hypothesis that the demographic, behavioral, and DTI data were normally distributed. We used Levene's and Mauchly' tests to verify, respectively, the homogeneity of variances for all the above-mentioned independent measures and the sphericity assumption for the behavioral indicator (i.e. CE) computed during the matching task.

Demographic and Clinical Data. Differences in age and gender between the 2 groups were assessed with the unpaired $t$-test and $\chi$ test, respectively.
Proprioceptive Matching Performance. To investigate significant difference during matching tasks, we performed a repeatedmeasures ANOVA with 1 between-subjects factor, i.e. Group (eRR-MS vs HS), and 2 within-subject factors, i.e. Task (CMA-R and IMA-R) and Position (DF7, REF, PF7, and PF14). When the sphericity assumption was rejected, we applied the Greenhouse-Geisser correction. Then, based on the ANOVA results, we used a Tukey's honest significant difference post hoc test to further investigate significant differences among the 4 target positions and an unpaired $t$-test to investigate specific differences between the 2 groups-HS and eRRMS patients - in each task. A Pearson correlation coefficient was used to assess the relationship between CE during CMA-R and $\mathrm{RMS}_{\mathrm{AP}}$ within the eRR-MS group.

Functional Magnetic Resonance Imaging. To strictly investigate position sense-related activity, we chose to contrast the matching tasks neural response with the purely active motor neural response, using 2-sample paired $t$-tests (IMA-R > ACTIVE-UNILAT, CMA-R > ACTIVE-BILAT), as performed in our previous study. ${ }^{27}$ Results were converted to $Z$-values, and then a threshold of $Z \geq 3.1$ for cluster formation and a significance threshold of $P=.0001$ (cluster corrected using Gaussian random field theory [GRFT]) were applied. In all the group-level analyses, we added age and gender as covariates for the HS group and age, gender, and disease duration for the eRR-MS group. To compare the activity during IMA-R and CMA-R between the HS and eRR-MS groups, we used the unpaired $t$-test. A threshold of $Z \geq 2.3$ for cluster formation and a significance threshold of $P=.01$ (cluster corrected using GRFT) were used.

The correlations between brain activations of eRR-MS patients (IMA-R and CMA-R) and the behavioral measure $\mathrm{CE}$ during the 2 matching tasks were modeled separately, with age, gender, and disease duration as covariates. A threshold of $Z \geq 2.3$ for cluster formation and using a significance threshold of $P=.01$ (cluster corrected using GRFT) were applied. We performed the same correlation analysis for the HS group, using age and gender as covariates.

In addition, to test the a priori hypothesis that there is a correlation between the balance parameter during quiet standing $\left(\mathrm{RMS}_{\mathrm{AP}}\right)$ and proprioceptive-related brain activity in the eRR-MS group, we used the same correlation analysis as above (for the CE). We selected the brain activity measured during CMA-R because for both fulfilling this task and maintaining balance during quiet standing, the proprioceptive representation of both legs is needed.

DTI Metrics and Behavioral Parameters. An unpaired $t$-test was used to assess differences in fractional anisotropy (FA) and mean diffusivity (MD) values of the CC between $\mathrm{HS}$ and eRR-MS. Moreover, partial correlation between the DTI metrics and CE during CMA-R was assessed in the 2 groups separately and in the HS + eRR-MS group, adding age and gender as covariates, using the Pearson correlation 
Table I. Demographic, Clinical, and Behavioral Data. ${ }^{a}$

\begin{tabular}{|c|c|c|}
\hline & Patients $(\mathrm{n}=24)$ & $\mathrm{HS}(\mathrm{n}=24)$ \\
\hline Age & $32.50 \pm 6.76$ & $30.75 \pm 4.54$ \\
\hline Gender & I7 Females; 7 males & I5 Females; 9 males \\
\hline Waterloo Scale score & $7.5 \pm 7.9$ & $11.82 \pm 4.91$ \\
\hline Disease duration (months) & $38.17 \pm 24.77$ & - \\
\hline EDSS (median; range) & I (0-3.5) & - \\
\hline s-FSS (median; range) & $0(0-2)$ & - \\
\hline CE CMA-R (degrees) & $10.89 \pm 4.56$ & $8.38 \pm 2.96$ \\
\hline CE IMA-R (degrees) & $6.33 \pm 2.53$ & $6.59 \pm 2.69$ \\
\hline $\mathrm{RMS}_{\mathrm{AP}}(\mathrm{mm})$ & $4.96 \pm 1.73$ & - \\
\hline T2-LV (mL) & $2.43 \pm 2.65$ & - \\
\hline TI-LV (mL) & $1.89 \pm 1.85$ & - \\
\hline CC FA & $0.64 \pm 0.03$ & $0.66 \pm 0.03$ \\
\hline CC MD $\left(\times 10^{-3} \mathrm{~mm}^{2} \mathrm{~s}^{-1}\right)$ & $0.77 \pm 0.02$ & $0.75 \pm 0.02$ \\
\hline
\end{tabular}

Abbreviations: CE, constant error; CMA-R, contralateral matching task performed with the right foot; EDSS, Expanded Disability Status Scale; FA, fractional anisotropy; $\mathrm{HS}$, healthy subject; IMA-R, ipsilateral matching task performed with the right foot; $M D$, mean diffusivity; $\mathrm{RMS}_{\mathrm{AP}}$, root mean square of the antero-posterior direction; s-FSS, sensory functional system score; TI-LV, lesion volume in TI-weighted sequence; T2-LV, lesion volume in T2-weighted sequence.

aMeans \pm SDs are reported if not otherwise specified.

coefficient. The latter test was also used to assess the relationship between diffusion metrics and the quiet standing parameter (i.e. $\mathrm{RMS}_{\mathrm{AP}}$ ) in the eRR-MS group.

\section{Results}

\section{Participants}

Demographic and clinical characteristics of all participants included in the analysis are reported in Table 1. The median sensory functional system score (s-FSS) in the eRR-MS group was 0 (range: $0-2$ ). The demographic data as well as the behavioral and DTI parameters were normally distributed $(P>.05$ in all cases). Age and gender did not significantly differ between the 2 groups: $t(46)=-1.05, P=.298$, and $\chi(1)=0.375, P=.540$ for age and gender, respectively.

\section{Behavioral Performance During Matching Tasks and During Quiet Standing}

The sphericity assumption was verified for both the group and task factors $(P>.05)$ but not for the positions factor $\left[\chi^{2}(2)=66.78, P<.001\right]$. Thus, for the latter case, we adopted the Greenhouse-Geisser correction $(\varepsilon=0.54)$. Both populations systematically overshot the target position. eRR-MS patients had a greater CE than HS during the CMA-R task, whereas during the IMA-R task, the 2 groups showed similar performance (Figure 2): Group $\times$ Task effect, $F(1,46)=7.26, P=.010$; Group effect, $F(1,46)=$ $1.98, P=.170$. Mean and SD CE values during CMA-R were $10.89^{\circ} \pm 4.56^{\circ}$ and $8.38^{\circ} \pm 2.96^{\circ}$ for eRR-MS and HS, respectively, with the unpaired $t$-test highlighting a significant difference $(t=2.26 ; P=.029)$. Mean and SD values for CE during IMA-R were $6.33^{\circ} \pm 2.53^{\circ}$ and $6.59^{\circ} \pm$ $2.69^{\circ}$ for eRR-MS and HS, respectively (unpaired $t$-test: $t=$ $-0.35 ; P=.730$ ). The $\mathrm{CE}$ during CMA-R was greater than during IMA-R for both populations [task effect: $F(1,46)=$ $38.31 ; P<.001]$.

The performance of eRR-MS related to postural control during quiet standing, i.e. the $\mathrm{RMS}_{\mathrm{AP}}$, is reported in Table 1 . RMS $_{\mathrm{AP}}$ significantly correlated with CE during CMA-R ( $r$ $=0.510 ; P=.015)$.

\section{Brain Activations During Matching and Motor Tasks in eRR-MS and HS}

Single-Group Analysis. Brain activity for both eRR-MS patients and HS groups is reported in Tables S1 and Table S2 for matching and motor tasks, respectively. See SI for a summary of brain regions involved.

\section{Within-Group Analysis in eRR-MS Patients}

(1) IMA-R > ACTIVE-UNILAT: the activation clusters involved the bilateral frontal areas, superior and inferior parietal lobule (SPL and IPL, respectively), primary somatosensory cortex (S1), right supramarginal gyrus (SMG), and left precuneus (Figure 3, Table S3).

(2) CMA-R > ACTIVE-BILAT: the contrast revealed several clusters covering parietal and frontal areas, in particular the bilateral postero-medial frontal cortex, right SPL, SMG, and S1, while the precuneus was activated bilaterally (Figure 3, Table S3).

(3) CMA-R vs IMA-R: see Table S4.

Between-Group Analysis. (1) Matching tasks: differences between eRR-MS patients and HS were detected between cortical activations during IMA-R but not CMA-R (see Table 2, Figure S1). (2) Motor tasks: see SI and Table S5. 

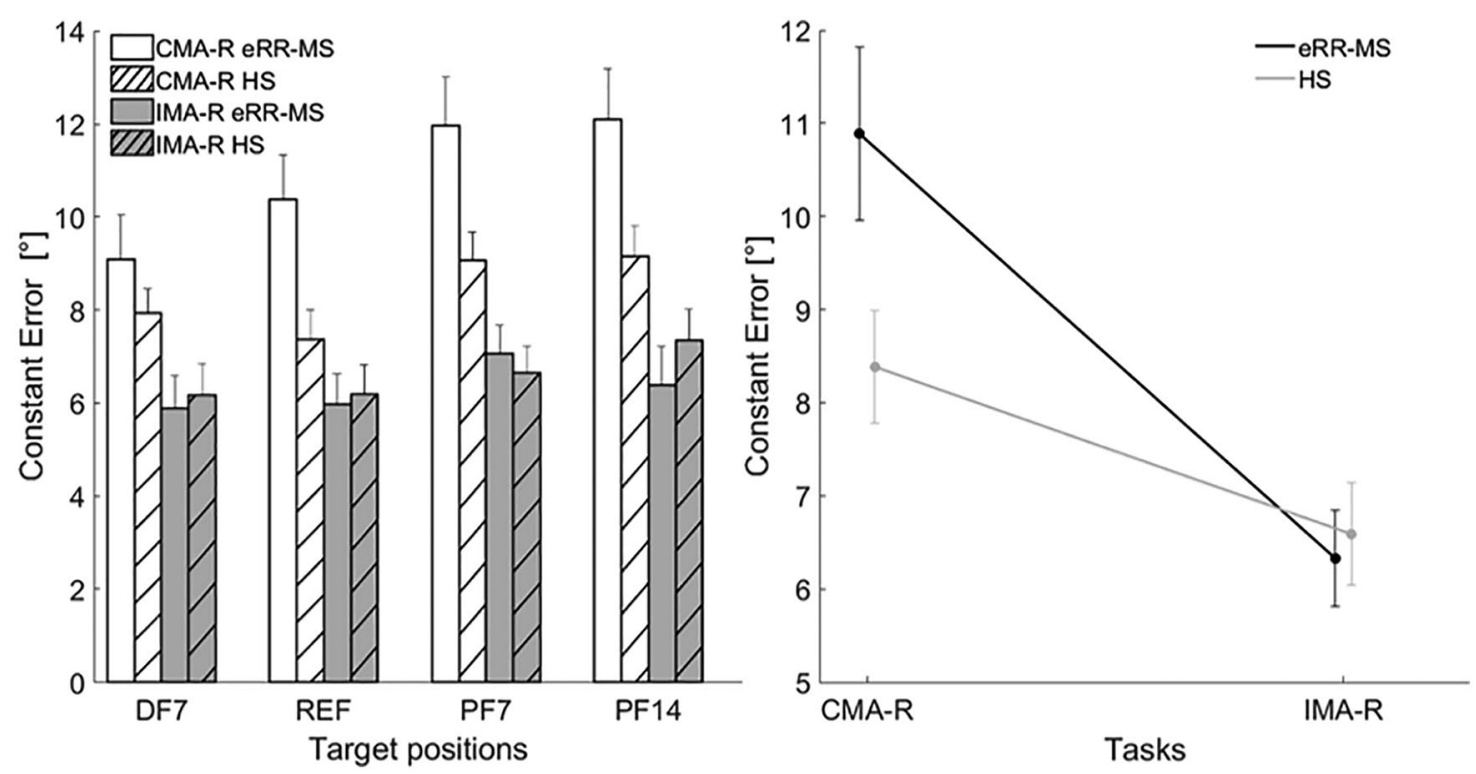

Figure 2. On the left: constant error for the 4 target angular positions (DF7, REF, PFI4, and PF7) presented during the matching tasks. Error bars represent the standard error (mean $\pm \mathrm{SE}$ ). On the right: mean group differences (eRR-MS vs HS, black and gray lines, respectively) in constant error for the 2 matching tasks (CMA-R and IMA-R). Filled circles and bars indicate means and SEs. Abbreviations: CMA-R, contralateral matching task performed with the right foot; eRR-MS, early relapsing-remitting multiple sclerosis; HS, healthy subjects; IMA-R, ipsilateral matching task performed with the right foot.

\section{Brain-Behavior Correlations}

In eRR-MS, during IMA-R, CE negatively correlated with brain activity at the level of the right S1 and SPL, whereas it positively correlated with brain activity at the level of the right frontal areas (Table S6, Figures 4A and 4B). In contrast, the CE during CMA-R positively correlated with an activation cluster at the level of the left motor and premotor area and S1 (Table S6, Figure 4C). Moreover, the RMS ${ }_{\mathrm{AP}}$ inversely correlated with cortical activation during CMA-R, in particular with activity at the level of the right SPL and IPL and primary motor cortex (Table S7, Figure 4D). No cortical or subcortical activation related to $\mathrm{CE}$ was found during either IMA-R or CMA-R in the HS group.

\section{Diffusion Tensor Metrics and Relationship With Behavioral Performance During CMA-R and During Quiet Standing}

Mean FA and MD values extracted from the CC (reported in Table 1) showed significant difference between eRR-MS patients and HS: unpaired $t$-test, $t(46)=3.015, P=.004$, and $t(46)=-3.522, P=.001$, respectively. Moreover, when considering both eRR-MS patients and HS, the FA and MD of the CC correlated with behavioral performance in terms of CE during CMA-R $(r=-0.298, P=.044$, and $r=0.335, P=.023$, respectively), but no significant correlation was found when the 2 groups were considered separately. No significant correlations were found between $\mathrm{FA}$ or $\mathrm{MD}$ of the $\mathrm{CC}$ and $\mathrm{RMS}_{\mathrm{AP}}$ for the eRR-MS group.

\section{Discussion}

In this work, we describe the behavioral and neural correlates of the position sense of the lower limb in eRR-MS patients as well as their relationship with balance performance during quiet standing. In summary, we found that eRR-MS patients presented a worse systematic matching error than controls during the contralateral but not during the ipsilateral task. However, compared with HS, eRR-MS patients showed a functional reorganization involving the parietal areas during the ipsilateral matching task whereas no differences between the 2 groups were found during CMA-R. Moreover, both the behavioral metric and the neural activity during CMA-R correlated with the performance during quiet standing. Lastly, the worse performance during the contralateral task correlated with the damage at the level of the CC.

\section{Position Sense Deficits and Their Neural Correlates During Lower Limb Matching Tasks}

In our study, both groups had a greater error during the contralateral than during the ipsilateral matching tasks, and this may be explained by the greater complexity of the former, 


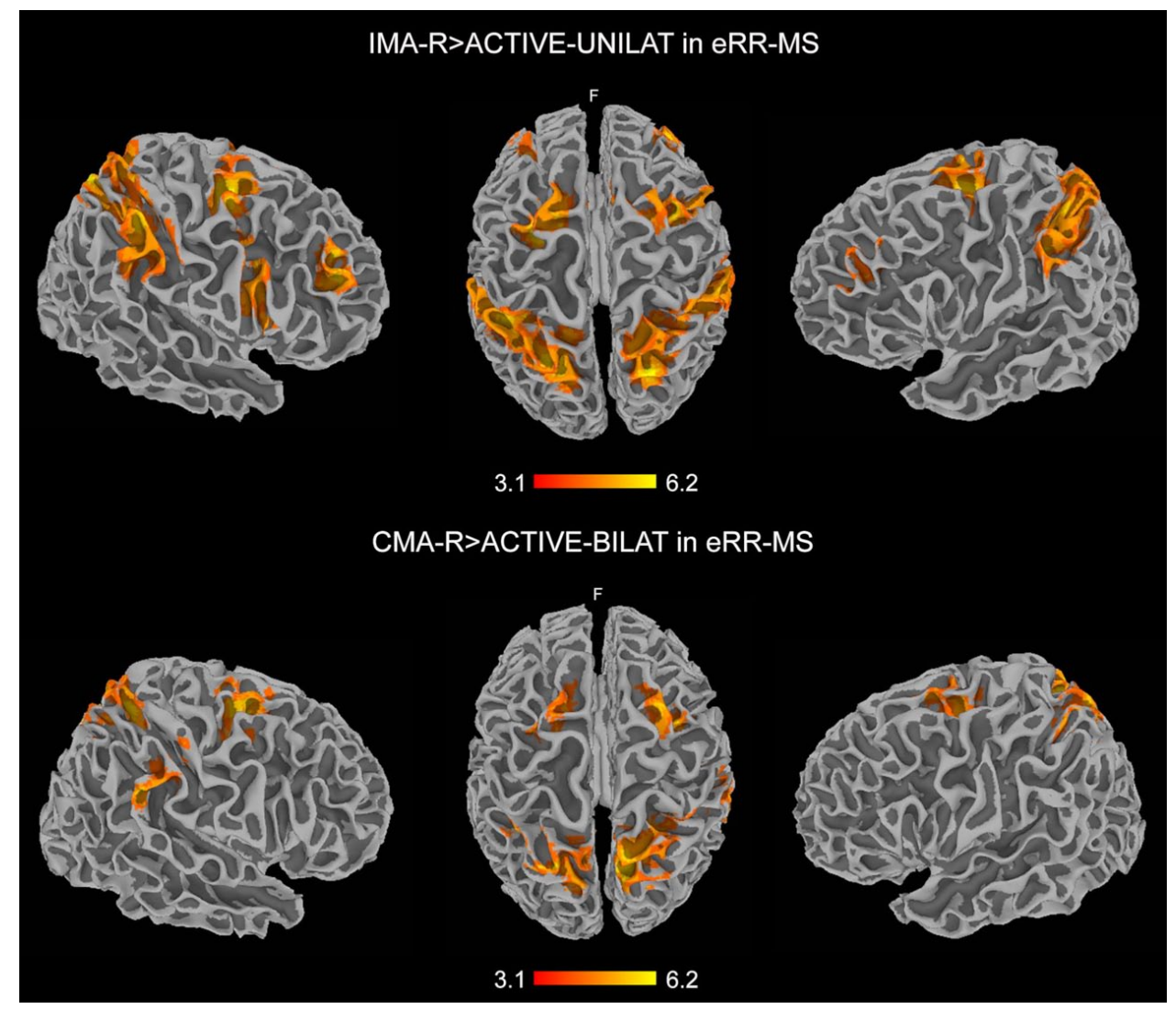

Figure 3. Clusters of activations resulting from the contrasts IMA-R $>$ ACTIVE-UNILAT (top) and CMA-R $>$ ACTIVE-BILAT (bottom), thus reflecting position sense neural correlates without the motor component of the task in the eRR-MS group. The results are cluster corrected for multiple comparisons $(Z \geq 3.1 ; P<.000 \mathrm{I})$ and are shown in MNI space. Note the cortical activity in the bilateral frontoparietal areas, more widespread during IMA-R $>$ ACTIVE-UNILAT.

Abbreviations: ACTIVE-UNILAT, unilateral active motor task; ACTIVE-BILAT, bilateral active motor task; CMA-R, contralateral matching task executed with the right foot; eRR-MS, early relapsing-remitting multiple sclerosis; IMA-R, ipsilateral matching task executed with the right foot.

Table 2. Differences in Brain Activation During IMA-R Between eRR-MS and HS.

\begin{tabular}{|c|c|c|c|c|c|c|}
\hline \multirow[b]{2}{*}{ Location } & \multirow{2}{*}{$\begin{array}{c}\text { CA } \\
\text { Location }\end{array}$} & \multicolumn{3}{|c|}{ MNI Coordinates } & \multirow[b]{2}{*}{ Z Score } & \multirow[b]{2}{*}{ Side } \\
\hline & & $x$ & $y$ & $z$ & & \\
\hline \multicolumn{7}{|c|}{ eRR-MS patients $>\mathrm{HS}$} \\
\hline IPL & Area hIP3 & -38 & -50 & 56 & 3.94 & $\mathrm{~L}$ \\
\hline IPL & Area hIP2 & -50 & -38 & 42 & 3.35 & $\mathrm{~L}$ \\
\hline PostCg & Area I & -30 & -42 & 66 & 3.2 & $\mathrm{~L}$ \\
\hline \multicolumn{7}{|c|}{$\mathrm{HS}>$ eRR-MS patients } \\
\hline MCC & & -4 & -40 & 38 & 4.67 & $\mathrm{~L}$ \\
\hline MCC & & 2 & -30 & 44 & 3.51 & $\mathrm{R}$ \\
\hline
\end{tabular}

Abbreviations: CA, cytoarchitectonic; eRR-MS, early relapsing-remitting multiple sclerosis; HS, healthy subject; IMA-R, ipsilateral matching task performed with the right foot; IPL, inferior parietal lobule; PostCg, postcentral gyrus; MCC, midcingulate cortex. which is associated with a higher likelihood of error. ${ }^{24,27}$ Moreover, eRR-MS patients had worse proprioceptive performance than HS during the contralateral but not during the ipsilateral matching task. This latter finding is relevant from a clinical point of view because it indicates that contralateral matching tasks can reveal subtle position sense deficits that are below the sensitivity of the standard clinical examination, as shown by the normal median s-FSS in our population of eRR-MS patients. Moreover, this result highlights the limits of the most used rating scale for assessing disability in MS - the EDSS - in properly evaluating deep sensation deficits. In fact, in the EDSS, position sense is scored together with other somatosensory modalities, thus making its impact on disability not easy to estimate. The introduction of a quantitative measure of proprioceptive deficits in clinical practice could help overcome this limit. 


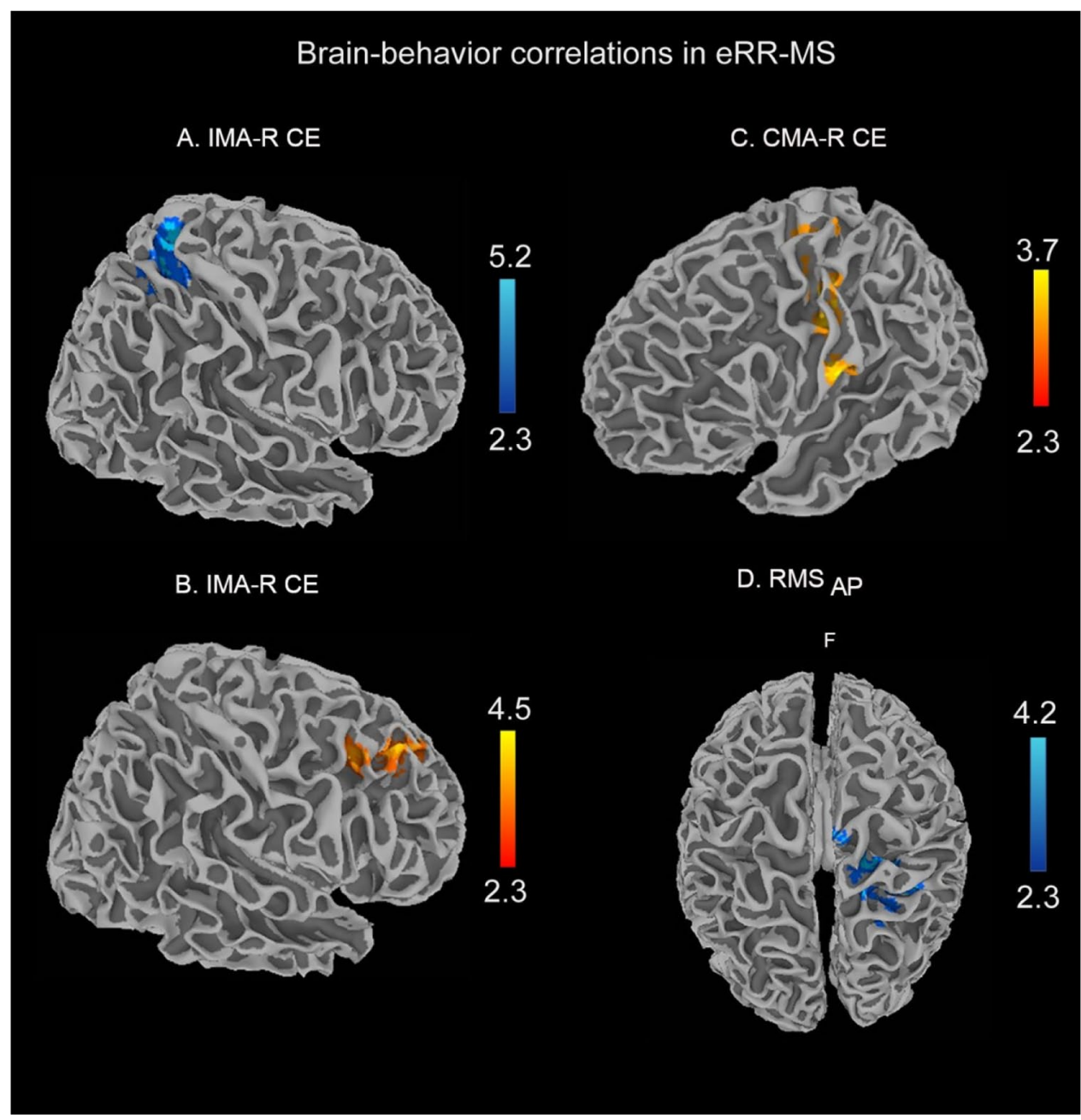

Figure 4. Clusters resulting from the correlation between BOLD signal and behavioral parameters during matching tasks (CMA-R and IMA-R CE) and during quiet standing $\left(\mathrm{RMS}_{\mathrm{AP}}\right)$ in the eRR-MS patient group. The results are cluster corrected for multiple comparisons $(Z \geq 2.3 ; P<.0 \mathrm{I})$ and are shown in $\mathrm{MNI}$ space. Brain activity during IMA-R presented both negative and positive correlations with the CE ( $A$ and $B$, respectively), whereas during CMA-R, only a positive correlation was found $(C)$. RMS $S_{A P}$ negatively correlated with brain activity during the CMA-R task (D).

Abbreviations: $C E$, constant error; CMA-R, contralateral matching task executed with the right foot; eRR-MS, early relapsing-remitting multiple sclerosis; IMA-R, ipsilateral matching task executed with the right foot; $\mathrm{RMS}_{\mathrm{AP}}$, root mean square of the anteroposterior oscillations of the center of pressure.

Although an indirect quantitative characterization of the proprioceptive impairment in patients with MS, using posturographic or electrophysiological measurements, has been suggested by previous studies, ${ }^{30,31}$ matching tasks allow a direct assessment of position sense and a quantification of the related deficits. ${ }^{24}$

As for the neural correlates of position sense, a predominant frontoparietal activity was detected in eRR-MS patients when the contrasts between matching tasks and pure motor tasks (IMA-R $>$ ACTIVE-UNILAT and CMA-R > ACTIVE-BILAT) were examined. Frontal areas play a role not only in sensory attentional and spatially oriented processing, but also in working memory processing, ${ }^{32-34}$ whereas parietal regions are involved in the processing of proprioceptive information, ${ }^{35}$ reorienting $^{36}$ and recognition of a matching position during visually guided tasks, ${ }^{37}$ and in the modulation of spinal motor output during movement preparation. ${ }^{38}$

Another relevant and novel finding of our study is the observation of a significant increase of brain activity in patients, compared with HS, during the ipsilateral matching task. This result, in combination with the absence of significant differences in behavioral performance between the two groups for this task, suggests an efficient supplying 
functional reorganization occurring during the performance of a simpler task, such as the ipsilateral matching task. Conversely, during the contralateral matching task, the modulation of functional activity was not accomplished or not sufficient, resulting in a compromised behavioral performance.

We found further evidence in support of the occurring functional reorganization. In fact, in eRR-MS, we found a negative correlation between CE during IMA-R and activity in the right parietal areas, but a positive correlation with right frontal areas. This result could further support a frontoparietal functional reorganization, at the level of the right hemisphere, underlying the lower rate of proprioceptive errors. Moreover, the positive correlation between CE during IMA-R and the right frontal cortex activation could imply the role of spatial attention ${ }^{39}$ in the execution of the task. In contrast, we could speculate that the positive correlation found between postcentral and precentral gyrus activation and CE during CMA-R represents an inefficient attempt to improve task performance.

No correlations were found in the HS group between brain functional activity and behavioral parameters during the matching tasks, further suggesting that the findings in the eRR-MS group are indicative of mechanisms of functional plasticity.

\section{Correlations With Behavioral Parameters During Quiet Standing}

In eRR-MS patients, the amplitude of the anteroposterior oscillations of the center of pressure $\left(\mathrm{RMS}_{\mathrm{AP}}\right)$ during quiet standing correlated positively with the errors (CE) during CMA-R, suggesting that the position sense deficits, measured with matching tasks, affect balance during quiet standing. The amplitude of the postural sway displayed also an inverse correlation with activations in different brain areas, and specifically at the level of the right parietal lobe, suggesting that this area, involved in the position sense processing, is related to the proprioception component undergirding balance performance.

\section{Behavioral Performance During Matching Tasks and CC Diffusion Tensor Metrics}

The behavioral performance during CMA-R was significantly associated with the mean FA and MD values of the $\mathrm{CC}$, leading us to speculate that during a bilateral task, such as the contralateral matching task, structural damage at the level of the CC prevents an efficient interhemispheric transfer of information as well as a functional cortical reorganization to compensate and guarantee a normal behavioral performance. Interestingly, results from previous studies seem to support this interpretation. In fact, a correlation between sensorimotor cortex functional connectivity and structural alterations of the CC, measured by means of DTI parameters, has already been reported in patients with eRRMS. ${ }^{40}$ Likewise, in a DTI study on HS, bimanual task performance in the absence of augmented visual feedback was significantly correlated with CC structural properties. ${ }^{41}$

We should acknowledge a few limitations of our study - first of all, the relatively small sample size of our patient group that included only those in the early stage of the disease. Although our approach and our findings are novel, they will have to be confirmed in larger populations of patients and at different stages of the disease. Admittedly, the lack of availability of postural control parameters in our control group limits the interpretation of the relationship observed between quiet standing behavioral measures and brain activity during matching tasks. However, the measurement of patients' postural control parameters has been instrumental to the assessment of the impact of our findings on balance. Moreover, our MRI protocol did not include sequences for the structural and functional assessment of spinal cord integrity, thus, precluding the investigation of the contribution of spinal cord abnormalities to proprioception processing. Lastly, although we performed this analysis on patients with low disability, the assessment of position sense by matching tasks on a sample of patients with moderate or severe disability could be influenced by their cognitive abilities, in particular in terms of attention. Therefore, cognitive assessment would be useful to exclude patients with attention impairment or to correct the results accordingly. At moderate or severe stages of the disease, other methods of proprioception assessment, such as the active movement extent discrimination apparatus, which requires less memory and attention, ${ }^{42}$ would be more appropriate.

\section{Conclusions}

Our study characterized the behavioral and neural correlates of position sense at the lower limbs in patients with early MS, revealing subtle deficits, which, nonetheless, were related to balance ability during quiet standing. Position sense processing relied on frontoparietal regions activation and a functional modulation occurred since the early stages of MS, possibly prevented by the CC damage for the contralateral task.

We believe that these findings are of great interest as they could provide a better insight into the pathophysiological mechanisms underlying disability in MS and facilitate the design of adequate protocols for detecting position sense deficits and for planning neurorehabilitation training that involves sensory functions.

\section{Authors' Note}

Data availability: Anonymized data are available on request from the corresponding authors. 


\section{Declaration of Conflicting Interests}

The author(s) declared no potential conflicts of interest with respect to the research, authorship, and/or publication of this article.

\section{Funding}

The author(s) disclosed receipt of the following financial support for the research, authorship, and/or publication of this article: Supported by a research fellowship FISM, Fondazione Italiana Sclerosi Multipla (Cod. 2017/B/2 and financed or cofinanced with the "5 per mille" public funding).

\section{ORCID iDs}

Giulia Bommarito iD https://orcid.org/0000-0003-2109-5443

Simona Schiavi (iD https://orcid.org/0000-0003-1641-186X

\section{References}

1. Lajoie Y, Teasdale N, Cole JD, et al. Gait of a deafferented subject without large myelinated sensory fibers below the neck. Neurology. 1996;47:109-115.

2. Bloem BR, Allum JHJ, Carpenter MG, Verschuuren JJ, Honegger F. Triggering of balance corrections and compensatory strategies in a patient with total leg proprioceptive loss. Exp Brain Res. 2002;142:91-107.

3. Gilman S. Joint position sense and vibration sense: anatomical organisation and assessment. J Neurol Neurosurg Psychiatry. 2002;73:473-477.

4. Dobson R, Giovannoni G. Multiple sclerosis - a review. Eur J Neurol. 2019;26:27-40.

5. Scalfari A, Neuhaus A, Degenhardt A, et al. The natural history of multiple sclerosis: a geographically based study 10: relapses and long-term disability. Brain. 2010;133(pt 7):1914-1929.

6. Jamali A, Sadeghi-Demneh E, Fereshtenajad N, Hillier S. Somatosensory impairment and its association with balance limitation in people with multiple sclerosis. Gait Posture. 2017;57:224-229.

7. Filli L, Sutter T, Easthope CS, et al. Profiling walking dysfunction in multiple sclerosis: characterisation, classification and progression over time. Sci Rep. 2018;8:4984.

8. Cameron MH, Lord S. Postural control in multiple sclerosis: implications for fall prevention. Curr Neurol Neurosci Rep. 2010;10:407-412.

9. Maggioni S, Melendez-Calderon A, van Asseldonk E, et al. Robot-aided assessment of lower extremity functions: a review. J Neuroeng Rehabil. 2016;13:72.

10. Rocca MA, Gatti R, Agosta F, et al. Influence of body segment position during in-phase and antiphase hand and foot movements: a kinematic and functional MRI study. Hum Brain Mapp. 2007;28:218-227.

11. Ciccarelli O, Toosy AT, Marsden JF, et al. Functional response to active and passive ankle movements with clinical correlations in patients with primary progressive multiple sclerosis. J Neurol. 2006;253:882-891.

12. Pantano P, Mainero C, Lenzi D, et al. A longitudinal fMRI study on motor activity in patients with multiple sclerosis. Brain. 2005;128(pt 9):2146-2153.
13. Goble DJ, Coxon JP, Van Impe A, et al. Brain activity during ankle proprioceptive stimulation predicts balance performance in young and older adults. J Neurosci. 2011;31:16344-16352.

14. Goble DJ, Coxon JP, Van Impe A, et al. The neural basis of central proprioceptive processing in older versus younger adults: an important sensory role for right putamen. Hum Brain Mapp. 2012;33:895-908.

15. Niam S, Cheung W, Sullivan PE, Kent S, Gu X. Balance and physical impairments after stroke. Arch Phys Med Rehabil. 1999;80:1227-1233.

16. Fanchamps MHJ, Gensicke H, Kuhle J, Kappos L, Allum JHJ, Yaldizli Ö. Screening for balance disorders in mildly affected multiple sclerosis patients. J Neurol. 2012;259: 1413-1419.

17. Prosperini L, Pozzilli C. The clinical relevance of force platform measures in multiple sclerosis: a review. Mult Scler Int. 2013;2013:756564.

18. Fling BW, Dutta GG, Schlueter H, Cameron MH, Horak FB. Associations between proprioceptive neural pathway structural connectivity and balance in people with multiple sclerosis. Front Hum Neurosci. 2014;8:814.

19. Prosperini L, Petsas N, Raz E, et al. Balance deficit with opened or closed eyes reveals involvement of different structures of the central nervous system in multiple sclerosis. Mult Scler. 2014;20:81-90.

20. Prosperini L, Sbardella E, Raz E, et al. Multiple sclerosis: white and gray matter damage associated with balance deficit detected at static posturography. Radiology. 2013;268: 181-189.

21. Peterson DS, Gera G, Horak FB, Fling BW. Corpus callosum structural integrity is associated with postural control improvement in persons with multiple sclerosis who have minimal disability. Neurorehabil Neural Repair. 2017;31:343-353.

22. Eliassen JC, Baynes K, Gazzaniga MS. Anterior and posterior callosal contributions to simultaneous bimanual movements of the hands and fingers. Brain. 2000;123(pt 12):2501-2511.

23. Lin F, Yu C, Liu Y, Li K, Lei H. Diffusion tensor group tractography of the corpus callosum in clinically isolated syndrome. AJNR Am J Neuroradiol. 2011;32:92-98.

24. Goble DJ. Proprioceptive acuity assessment via joint position matching: from basic science to general practice. Phys Ther. 2010;90:1176-1184.

25. Elias LJ, Bryden MP, Bulman-Fleming MB. Footedness is a better predictor than is handedness of emotional lateralization. Neuropsychologia. 1998;36:37-43.

26. Thompson AJ, Banwell BL, Barkhof F, et al. Diagnosis of multiple sclerosis: 2017 revisions of the McDonald criteria. Lancet Neurol. 2018;17:162-173.

27. Iandolo R, Bellini A, Saiote C, et al. Neural correlates of lower limbs proprioception: an fMRI study of foot position matching. Hum Brain Mapp. 2018;39:1929-1944.

28. Baratto L, Morasso PG, Re C, Spada G. A new look at posturographic analysis in the clinical context: sway-density versus other parameterization techniques. Motor Control. 2002;6:246-270.

29. Severini G, Straudi S, Pavarelli C, et al. Use of Nintendo Wii Balance Board for posturographic analysis of multiple sclerosis patients with minimal balance impairment. J Neuroeng Rehabil. 2017;14:19. 
30. Thoumie P, Mevellec E. Relation between walking speed and muscle strength is affected by somatosensory loss in multiple sclerosis. J Neurol Neurosurg Psychiatry. 2002;73:313-315.

31. Fritz NE, Marasigan RER, Calabresi PA, Newsome SD, Zackowski KM. The impact of dynamic balance measures on walking performance in multiple sclerosis. Neurorehabil Neural Repair. 2015;29:62-69.

32. Shulman GL, Pope DLW, Astafiev SV, McAvoy MP, Snyder AZ, Corbetta M. Right hemisphere dominance during spatial selective attention and target detection occurs outside the dorsal frontoparietal network. J Neurosci. 2010;30:3640-3651.

33. Noyce AL, Cestero N, Michalka SW, Shinn-Cunningham BG, Somers DC. Sensory-biased and multiple-demand processing in human lateral frontal cortex. $J$ Neurosci. 2017;37:8755-8766.

34. du Boisgueheneuc F, Levy R, Volle E, et al. Functions of the left superior frontal gyrus in humans: a lesion study. Brain. 2006;129(pt 12):3315-3328

35. Keysers C, Kaas JH, Gazzola V. Somatosensation in social perception. Nat Rev Neurosci. 2010;11:417-428.

36. Schurz M, Tholen MG, Perner J, Mars RB, Sallet J. Specifying the brain anatomy underlying temporo-parietal junction activations for theory of mind: a review using probabilistic atlases from different imaging modalities. Hum Brain Mapp. 2017;38:4788-4805.

37. Limanowski J, Blankenburg F. Integration of visual and proprioceptive limb position information in human posterior parietal, premotor, and extrastriate cortex. $J$ Neurosci. 2016;36:2582-2589.

38. Mackenzie TN, Bailey AZ, Mi PY, Tsang P, Jones CB, Nelson AJ. Human area 5 modulates corticospinal output during movement preparation. Neuroreport. 2016;27:1056-1060.

39. Jueptner M, Stephan KM, Frith CD, Brooks DJ, Frackowiak RS, Passingham RE. Anatomy of motor learning. I. Frontal cortex and attention to action. J Neurophysiol. 1997;77:13131324.

40. Lowe MJ, Beall EB, Sakaie KE, et al. Resting state sensorimotor functional connectivity in multiple sclerosis inversely correlates with transcallosal motor pathway transverse diffusivity. Hum Brain Mapp. 2008;29:818-827.

41. Gooijers J, Caeyenberghs K, Sisti HM, et al. Diffusion tensor imaging metrics of the corpus callosum in relation to bimanual coordination: effect of task complexity and sensory feedback. Hum Brain Mapp. 2013;34:241-252.

42. Han J, Waddington G, Adams R, Anson J, Liu Y. Assessing proprioception: a critical review of methods. J Sport Health Sci. 2016;5:80-90. 